\title{
A-20
}

\section{RIEGO DEFICITARIO CONTROLADO EN MELOCOTONERO CATERINA}

Ruiz Gómez, J.C., Temnani Rajjaf, A., Blaya Ros, P.J., Fernández Bastida, I, Cañavate Sastre, M.D., López Guerrero, M.C., Pérez Abellán, M.A., Morales Navarro, A., Navarrete

Martínez, S., de la Rosa Sánchez, J.M., Conesa Saura, M.R., Pérez-Pastor, A. *(P)

Universidad Politécnica de Cartagena (UPCT). Dpto. Producción Vegetal. Paseo Alfonso XIII, 48. 30203. Cartagena (Murcia). alex.perez-pastor@upct.es

\section{1- Introducción}

En zonas áridas como la región de Murcia los agricultores se ven obligados a regar por debajo de las necesidades hídricas del cultivo.

Dentro de las estrategias de riego deficitario, el Riego Deficitario Controlado (RDC) permite reducir los aportes hídricos en periodos fenológicos del cultivo (no críticos) donde el déficit hídrico no afecta negativamente a la calidad y cantidad de la cosecha, cubriendo plenamente las necesidades durante el resto del ciclo. Los periodos no críticos del melocotonero son las fases de crecimiento del fruto I y II y la poscosecha.

El objetivo de este trabajo fue evaluar el efecto del riego deficitario en las fases I y II de crecimiento del fruto del melocotonero en: i) los indicadores del estado hídrico de la planta; ii) el crecimiento vegetativo; iii) la producción y iv) la calidad de la cosecha.

\section{2- Material y métodos}

El estudio se realizó entre febrero y agosto de 2015 en la finca comercial "Los Periquitos" situada en Molina de Segura, Murcia (38 $\left.9^{\prime} \mathrm{N} ; 1^{\circ} 14^{\prime} \mathrm{S}\right)$. Los melocotoneros (Prunus persica (L.) Batsch) variedad Caterina tenían 7 años de edad al inicio del experimento y estaban colocados a un marco de plantación de $5 \times 3.5 \mathrm{~m}$.

Se establecieron dos tratamientos de riego: i) Control $\left(\mathrm{T}_{\mathrm{CTL}}\right)$ regado al $100 \%$ de la evapotranspiración de cultivo (ETc) durante todo el periodo; y Riego Deficitario Controlado $\left(T_{R D C}\right)$ regado como $T_{C T L}$ excepto en la fases I y II de crecimiento del fruto en las que se regó la mitad.

Se determinó el estado hídrico de la planta a partir de medidas de: i) potencial hídrico de tallo al mediodía $\left(\Psi_{\mathrm{T}}\right)$; Fluctuaciones de diámetro de tronco, a partir de las cuales se calculó el máximo diámetro de tronco (MXDT) y el mínimo diámetro de tronco (MNDT) y se determinó la máxima contracción diaria (MCD=MXDT-MNDT) y la tasa de crecimiento diaria $\left(\mathrm{TCD}=\mathrm{MXDT} \mathrm{T}_{2}-\mathrm{MXDT}_{1}\right)$.

Se evaluó el crecimiento vegetativo y del fruto a partir de medidas puntuales de longitud de brotes y diámetro ecuatorial de frutos. Se contabilizó la producción y el número de frutos recolectados. El tamaño medio del fruto se determinó como la relación entre la producción y el número de frutos de cada árbol.

Se evaluaron parámetros de calidad como la firmeza, el contenido de sólidos solubles totales, la acidez, el índice de madurez y la jugosidad.

\section{3.- Resultados}

El riego aplicado durante el periodo estudiado fue 3666 y $2878 \mathrm{~m}^{3} \cdot \mathrm{ha}^{-1}$ para $\mathrm{T}_{\text {CTL }}$ y $\mathrm{T}_{\mathrm{RDC}}$, respectivamente, lo que supuso una disminución de un $21.5 \%$ en $\mathrm{T}_{\mathrm{RDC}}$. 
Los indicadores de estado hídrico de planta, MCD, TCD y $\Psi_{\mathrm{T}}$, no presentaron diferencias claras entre tratamientos durante el periodo de déficit hídrico lo que puede ser debido a la hora de inicio del riego (impuesta por el agricultor) ya que éste empezaba a las 10 de la mañana y duraba entre 3 y 5 horas.

El crecimiento acumulado del tronco (MXDT) presentaba valores significativamente inferiores en $\mathrm{T}_{\mathrm{RDC}}$ durante el periodo de déficit hídrico para recuperarse tras la reanudación del riego en plenitud.

$\mathrm{T}_{\mathrm{RDC}}$ presentó diámetros ecuatoriales de fruto significativamente inferiores a los de $T_{C T L}$ al final del periodo de déficit. Tras el déficit hídrico los frutos de $T_{R D C}$ experimentaron un crecimiento compensatorio que permitió que los dos tratamientos se igualaran antes de la recolección. No se han encontrado diferencias significativas ni en producción, ni en ninguno de los parámetros de calidad estudiados.

\section{4.- Conclusiones}

La estrategia de riego deficitario controlado estudiada ha permitido disminuir el aporte hídrico en $800 \mathrm{~m}^{3} \cdot \mathrm{ha}^{-1}$ sin generar mermas en la cantidad y calidad de la cosecha e incrementando la eficiencia en el uso del agua de riego. 